\title{
ISOGENY RESTRICTIONS OF IRREDUCIBLE ADMISSIBLE REPRESENTATIONS ARE FINITE DIRECT SUMS OF IRREDUCIBLE ADMISSIBLE REPRESENTATIONS ${ }^{1}$
}

\author{
ALLAN J. SILBERGER
}

ABSTRACT. This paper proves that the isogeny restriction of an irreducible admissible representation of a connected reductive algebraic $p$-adic group decomposes as a finite direct sum of irreducible admissible representations.

Let $\tilde{G} \stackrel{\tilde{\phi}}{\rightarrow} \tilde{G}^{*} \rightarrow 1$ be a central $\Omega$-isogeny of connected reductive $\Omega$-groups, where $\Omega$ is a nonarchimedean local field. Then $\tilde{\phi}$ induces a continuous homomorphism $\phi: G \rightarrow G^{*}$ of the corresponding groups of $\Omega$-points and $\phi(G)$ is a closed subgroup of $G^{*}$ such that $G^{*} / \phi(G)$ is compact and abelian.

The purpose of this note is to prove the following:

THEOREM. Let $\pi^{*}$ be an irreducible admissible representation of $G^{*}$. Then $\pi=\pi^{*} \circ \phi$ decomposes as a finite direct sum of irreducible admissible representations of $G$.

We thank the referee for pointing out that our original proof of the above theorem could be shortened and simplified.

The proof. In the following capital letters denote groups of $\Omega$-points of $\Omega$-groups; a tilde over the letter indicates the algebraic group.

LEMMA 1. If $\pi^{*}$ is supercuspidal, then $\pi$ is supercuspidal and finitely generated.

Proof. Since $\pi^{*}$ is smooth and irreducible and since $G^{*} / \phi(G)$ is compact, $\pi$ is finitely generated. Since every unipotent element of $G^{*}$ lies in the image of $\phi$ and since $\pi^{*}$ is supercuspidal, $\pi$ is supercuspidal (i.e., admissible, too, as follows from [1, Theorem 6] and [2, Lemma 1.11.4]).

LEMMA 2. Let $\pi^{*}$ be a finitely generated admissible representation of $G^{*}$. Then $\pi$ is also finitely generated and admissible.

Proof. If $0 \rightarrow V_{1} \rightarrow V_{2} \rightarrow V_{3} \rightarrow 0$ is an exact sequence of smooth $G^{*}$ modules, then $V_{2}$ is admissible if and only if $V_{1}$ and $V_{3}$ are. An admissible

Received by the editors May 24, 1978.

AMS (MOS) subject classifications (1970). Primary 22E50.

Key words and phrases. Isogeny, admissible representation, reductive group.

${ }^{1}$ Research partially supported by the National Science Foundation. 
representation is finitely generated if and only if it has finite length [2, Theorem 5.4.1.6]. Thus, it suffices to consider the case in which $\pi^{*}$ is irreducible.

In this case, by a theorem of Jacquet, one can find a $p$-subgroup $P^{*}=$ $M^{*} N^{*}$ of $G^{*}$ and an irreducible supercuspidal representation $\sigma^{*}$ of $M^{*}$ such that $\pi^{*}$ is imbedded in $\operatorname{Ind}_{P^{*}}^{G^{*}} \sigma^{*}$. Let $P=\phi^{-1}\left(P^{*}\right)$. Then $P$ is a $p$-subgroup of $G$. There is a Levi decomposition $\tilde{P}=\tilde{M} \tilde{N}$ for $\tilde{P}$ such that $\tilde{\phi} \mid \tilde{P}$ induces an isogeny $\tilde{M}^{\tilde{\phi}_{M}} \rightarrow \tilde{M}^{*} \rightarrow 1$. Since representatives for $G^{*} / \phi(G)$ may be chosen in $M^{*}$ and since both $G^{*}$ and $G$ have Iwasawa decompositions, it is clear that $\left(\operatorname{Ind}_{P *}^{G^{*}} \sigma^{*}\right) \circ \phi=\operatorname{Ind}_{P}^{G} \sigma$, where $\sigma=\sigma^{*} \circ \phi_{M}$. Since the functor $\operatorname{Ind}_{P}^{G}$ sends admissible $M$-modules of finite type to admissible $G$-modules of finite type [2, Theorem 5.4.4.1], this lemma follows from the preceding one.

Leмma 3. If $\pi^{*}$ is an irreducible admissible representation of $G^{*}$, then $\pi$ is completely reducible.

Proof. By Lemma 2, $\pi$ is admissible of finite type. Let $\pi_{1}$ be any irreducible subrepresentation of $\pi$. Then, for any $g \in G^{*}, \pi^{*}(g)$ intertwines $\pi_{1}$ with an irreducible subrepresentation ${ }^{8} \pi_{1}$ of $\pi$, where

$$
s_{\pi_{1}}(x)=\pi_{1}\left(g x g^{-1}\right) \text { for all } x \in G \text {. }
$$

Thus, $\pi^{*}$ stabilizes the maximum completely reducible subrepresentation of $\pi$, so $\pi$ is itself completely reducible.

\section{REFERENCES}

1. Harish-Chandra, Harmonic analysis on reductive p-adic groups, (Lectures at the Institute for Advanced Study, Fall, 1969, notes by G. van Dijk), Lecture Notes in Math., vol. 162, SpringerVerlag, Berlin and New York, 1970.

2. A. J. Silberger, Introduction to harmonic analysis on reductive p-adic groups, based on lectures by Harish-Chandra at the Institute for Advanced Study, 1971-1973, Princeton Univ. Press Lecture Note Series (to appear).

Department of Mathematics, Cleveland State University, Cleveland, OHo 44115 